\title{
Multi-Band Nonlinear Schrödinger Equation for Efficient Simulation of Parametric Optical Amplifiers and Oscillators
}

\author{
Ioannis Begleris and Peter Horak* \\ Optoelectronics Research Centre, University of Southampton, Southampton SO17 1BJ, United Kingdom \\ *e-mail:peh@orc.soton.ac.uk
}

Keywords: Nonlinear fibre optics; parametric amplifiers; parametric oscillators; numerical simulation.

For the theoretical description of laser pulse propagation in optical fibres in parameter regimes where linear dispersive and nonlinear optical effects both play an important role in the pulse dynamics, the generalised nonlinear Schrödinger equation (GNLSE) has long been established as the appropriate theoretical framework. In such simulations, uniform grids in the time and frequency domains need to be chosen that are related to each other by the properties of the Fourier transform. Thus, when the system dynamics includes long pulses with widely different carrier frequencies, a huge number of grid points are required to resolve the light field both spectrally and temporally making the simulations slow and inefficient. Such a situation typically occurs in fibre optical parametric amplifiers (OPA) and oscillators (OPO) where a narrow-band pump pulse interacts with narrow-band signal and idler pulses that can be separated in wavelength by $100 \mathrm{~s}$ of nm [1] or even $>1 \mu \mathrm{m}$ [2].

Here we present a solution to this problem by developing a theoretical and numerical framework that deals with such a situation while still including all the relevant Kerr and Raman nonlinear effects such as self-phase modulation, cross phase modulation, parametric and Raman gain, and arbitrary chromatic dispersion profiles. To this end, we split the GNLSE into distinct narrow frequency bands around the relevant carrier frequencies and neglect frequency regions without significant spectral power density. The resulting set of coupled nonlinear Schrödinger equations for the individual frequency bands (e.g. the pump, signal, and idler pulses in an OPA/OPO), which we term the "banded nonlinear Schrödinger equation" (BNLSE), employs grid sizes that can be $10 \mathrm{~s}$ or $100 \mathrm{~s}$ of times smaller than those required for the full GNLSE with a corresponding reduction in required computer memory and execution times while maintaining the same accuracy of results.

We numerically test this novel BNLSE simulation platform and compare the results with the GNLSE as well as with results from the simplified coupled amplitude equations that are currently the main numerical method to investigate fibre OPAs. Our results clearly show the accuracy and efficiency of the BNLSE, irrespective of whether the signal band of an OPA lies inside or outside of the Raman gain bandwidth.

We finally apply the BNLSE to simulate a fibre OPO [3] where the output of one roundtrip of the signal pulse in a fibre loop is used as the input for the subsequent roundtrip after mixing with a new pump pulse. These simulations require up to 1000 s of roundtrip simulations to find the steady state of the OPO [4] and are therefore particularly sensitive to efficient numerical implementation. We simulate OPO thresholds versus system parameters such as the offset of the pump wavelength from the zero-dispersion wavelength of the fibre and analyse the output power noise figure.

These initial results of the BNLSE are highly encouraging and align well with the current interest in parametric amplification across a range of fields, from mid-IR laser sources for spectroscopy to wavelength conversion [5] and phase sensitive signal processing [6] in coherent optical telecommunication systems.

\section{ACKNOWLEDGEMENTS}

This work was supported by the U.K. Engineering and Physical Sciences Research Council (EPSRC) through the Centre for Doctoral Training in Next Generation Computational Modelling (grant EP/L015382/1).

\section{REFERENCES}

[1] P. Dupriez et al.: Efficient white light generation in secondary cores of holey fibers, Opt. Expr., vol. 15, pp. 3729-3736, Apr. 2007.

[2] M.I.M. Abdul Khudus et al.: Phase matched parametric amplification via four-wave mixing in optical microfibers, Opt. Lett., vol. 41, pp. 761-764, Feb. 2016.

[3] E.A. Zlobina, S.I. Kablukov, and S.A. Babin: High-efficiency CW all-fiber parametric oscillator tunable in 0.92-1 $\mu \mathrm{m}$ range, Opt. Expr., vol. 23, pp. 833-838, Jan. 2015.

[4] I. Begleris and P. Horak: Cascade simulations of unidirectional fiber optical parametric oscillators, in Proc. NUSOD 2017, Copenhagen, Denmark, Jul. 2017, pp. 169-170.

[5] F. Parmigiani et al.: All-optical mode and wavelength converter based on parametric processes in a threemode fiber, Opt. Expr., vol. 25, pp. 33602-33609, Dec. 2017.

[6] F. Parmigiani et al.: Polarization-assisted phase-sensitive processor, J. Lightw. Technol., vol. 33, pp. 11661174, Mar. 2015. 